\title{
A distinct cutaneous leishmaniasis lesion on the tip of the patient's nose: A visual warning for European colleagues
}

\author{
Funda Tamer', Mehmet Eren Yuksel ${ }^{2}$
}

${ }^{1}$ Department of Dermatology, Medical Park Hospital, Ankara, Turkey, ${ }^{2}$ Devrek State Hospital, Department of General Surgery, Zonguldak, Turkey

Corresponding author: Dr. Funda Tamer, E-mail: fundatmr@yahoo.com

Sir,

An 18-year-old Caucasian male presented with a six-month history of non-healing wound on his nose. The patient admitted that the lesion started as a small erythematous papule and it increased in size gradually. Thereafter, the lesion became ulcerated. It was painless, however bleeding occured when the patient scratched the lesion on the tip of his nose. The past medical history was unremarkable. The patient was living in a rural area of eastern Anatolia in Turkey. The physical examination revealed an erythematous, indurated, crusted plaque on the tip of the nose (Figs. 1 and 2). A skin biopsy was performed to reach a definitive diagnosis. The histopathological examination revealed cutaneous leishmaniasis. The patient was treated with intralesional meglumine antimoniate three times a week and cryotherapy every two weeks untill the lesion was completely healed.

Cutaneous leishmaniasis is a parasitic disease which clinically presents with erythema, papule, nodule and ulcerative skin lesions. The causative agent of cutaneous leishmaniasis is the leishmania parasite which has several species. The leishmania parasites are spread by the bite of infected sandflies. Therefore, the leishmania lesions usually occur on the site of the sandfly bites including face, hands and feet [1]. It has been suggested that approximately 1.71 billion people are at risk for cutaneous leishmaniasis in the world [2]. The presence of sandflies, climate factors like temperature, unawareness of the disease, poor personal protection, poor housing and environmental

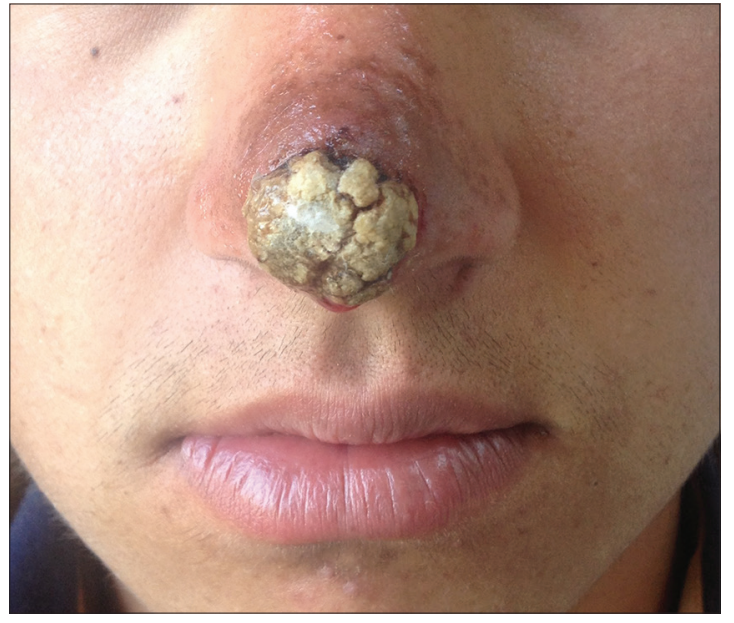

Figure 1: Cutaneous leishmaniasis: An erythematous, indurated, crusted plaque on the tip of the patient's nose.

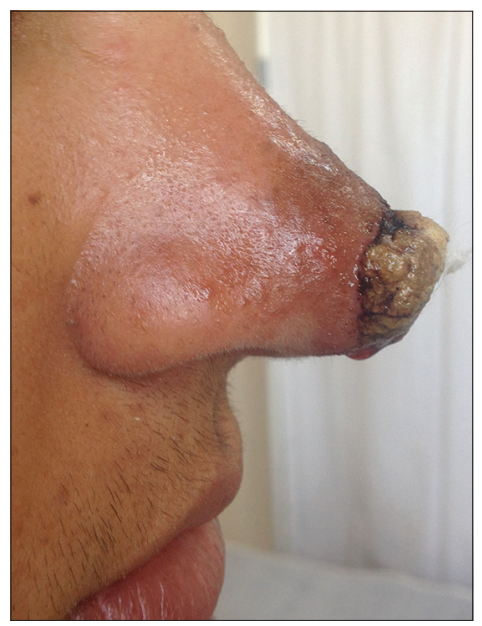

Figure 2: The right lateral view of the cutaneous leishmaniasis lesion on the patient's nose.

\footnotetext{
How to cite this article: Tamer F, Yuksel ME. A distinct cutaneous leishmaniasis lesion on the tip of the patient's nose: A visual warning for European colleagues. Our Dermatol Online. 2017;8(1):108-109.

Submission: 27.04.2016; Acceptance: 11.06.2016

DOI:10.7241/ourd.20171.31
} 
conditions are the factors which affect the incidence and distribution of cutaneous leishmaniasis [2]. Cutaneous leishmaniasis occurs worldwide, however, most cases ocur in Afghanistan, Algeria, Brazil, Pakistan, Peru, Saudi Arabia, and Syria [1].

Cutaneous leishmaniasis is endemic in Syria and increased incidence of the disease has been reported between 1997 and 2011 [3]. Recently, civil war in Syria led massive displacement of people. Therefore, the number of cutaneous leishmaniasis cases increased significantly in neighbouring countries like Turkey, Jordan and Lebanon [3]. Alawieh et al. reported that 96.6\% of the all new cases in Lebanon in 2013 occured among Syrian refugees [4].

Cutaneous leishmaniasis is endemic in southeastern Anatolia, eastern Anatolia and eastern mediterranean regions of Turkey. Inci et al. investigated the effect of Syrian war on prevalence of cutaneous leishmaniasis in southeastern Anatolia. They evaluated 110 patients who were diagnosed with cutaneous leishmaniasis between 2011 and 2014. 69\% of the patients were Syrian refugees. Inci et al. reported that there was a relationship between increased incidence of cutaneous leishmaniasis and increased number of Syrian refugees from highly endemic areas [5].

Moreover, refugees from countries where cutaneous leishmaniasis is endemic may spread the infection to non-endemic regions including European countries. For this reason, health care providers in Europe should be aware of the diagnosis and management of cutaneous leishmaniasis. Therefore, we would like to share a case of cutaneous leishmaniasis with distinct clinical features with our European colleagues.

\section{REFERENCES}

1. Reithinger R, Dujardin JC, Louzir H, Pirmez C, Alexander B, Brooker S. Cutaneous leishmaniasis. Lancet Infect Dis. 2007;7:581-96.

2. Haouas N. Estimations of cutaneous leishmaniasis burden: a constant challenge. Lancet Infect Dis. 2016. pii: S14733099(16)00058-X.

3. Haddad N, Saliba H, Altawil A, Villinsky J, Al-Nahhas S. Cutaneous leishmaniasis in the central provinces of Hama and Edlib in Syria: Vector identification and parasite typing. Parasit Vectors. 2015;8:524.

4. Alawieh A, Musharrafieh U, Jaber A, Berry A, Ghosn N, Bizri AR. Revisiting leishmaniasis in the time of war: the Syrian conflict and the Lebanese outbreak. Int J Infect Dis. 2014;29:115-9.

5. Inci R, Ozturk P, Mulayim MK, Ozyurt K, Alatas ET, Inci MF. Effect of the Syrian civil war on prevalence of cutaneous leishmaniasis in southeastern Anatolia, Turkey. Med Sci Monit. 2015;21:2100-4.

Copyright by Funda Tamer, et al. This is an open access article distributed under the terms of the Creative Commons Attribution License, which permits unrestricted use, distribution, and reproduction in any medium, provided the original author and source are credited.

Source of Support: Nil, Conflict of Interest: None declared. 